\title{
Computing Approximate Blocking Probabilities in Wavelength Routed All-Optical Networks with Limited-Range Wavelength Conversion
}

\author{
Tushar Tripathi and Kumar N. Sivarajan \\ Electrical Communication Engineering Department \\ Indian Institute of Science \\ Bangalore 560012 , India \\ e-mail: tushart, kumareece. iisc.ernet. in
}

\begin{abstract}
In this paper, we propose a method to calculate the average blocking probability in all-optical networks using limited-range wavelength conversion. Previous works have shown that there is a remarkable improvement in blocking probability while using limited-range wavelength conversion [2], [3] but these analytical models were either for a path [2] or for a mesh-torus network [3]. Using a graph-theoretical approach, we extend Birman's model [4] for no wavelength conversion and derive an analytical expression to compute the blocking probabilities in networks with limited-range wavelength conversion for fixed routing. The proposed analytical model is a generalization of Birman's model and is applicable to any network topology. We consider the case where an incoming wavelength can be converted to $d$ adjacent outgoing wavelengths on either side of the input wavelength, in addition to the input wavelength itself, where $d$ is the degree of conversion. Using this model we demonstrate that the performance improvement obtained by full wavelength conversion over no wavelength conversion can almost be achieved by using limited wavelength conversion with the degree of conversion, $d$, being only 1 or 2 . In a few example networks we considered, for blocking probabilities up to a few percent, the carried traffic with limited conversion degree $d=2$ was almost equal to the carried traffic for full wavelength conversion.

The results obtained show that significant improvements in blocking performance can be obtained by providing limited-range wavelength conversion of small degree within the network.
\end{abstract}

\section{INTRODUCTION}

All-optical networks have attracted a lot of interest in the networking community in the past few years. The reason for this is that a single fiber provides an enormous bandwidth $(25 \mathrm{THz})$, low loss $(0.2 \mathrm{~dB} / \mathrm{Km})$ and very low bit error rate $\left(10^{-9}-10^{-15}\right)$. In all-optical networks the data remain in the optical domain throughout their path except at the ends. Such paths are termed as lightpaths. Lightpaths can be viewed as huge bandwidth pipes into which we can pump data at different bit rates using different modulation schemes and different protocols. Therefore all-optical networks provide significant advantages in terms of transparency, future-proofness and reduced processing. The currently favoured technology to tap this huge bandwidth of optical fiber is wavelength division multiplexing (WDM). In WDM networks, the optical spectrum is divided into many different channels and each channel corresponds to a different wavelength which can operate at the peak electronic speed. Optical networks can be classified in two broad categories according to their architecture: (1) Broadcast and select networks, and (2) Wavelength routed networks. Broadcast and select networks, though simpler in terms of ar-

This research was supported by a grant from the Department of Science and Technology, Government of India. chitecture and management, do not allow wavelength reuse. Using wavelength routed WDM networks, we can reuse the wavelength provided no two lightpaths sharing a link are assigned the same wavelength. Therefore, WDM wavelength routed networks are scalable and better suited for WAN applications.

To maximize the number of connections in WDM wavelength routed networks, a number of wavelength routing and assignment algorithms have been proposed and analyzed. In all these studies, it is clearly seen that full wavelength conversion does better than no wavelength conversion [1], [4], [10] and this gain in advantage using full wavelength conversion depends on a host of network parameters such as topology and connectivity [6] and other factors such as the number of wavelengths per fiber, number of fibers per link and routing and wavelength assignment algorithms.

In networks using full wavelength conversion a call is accepted if on all the links on its route there is at least one free wavelength. With no wavelength conversion a call is accepted on a route if there exists at least one wavelength which is simultaneously free on all the links of that route. This constraint is known as the wavelength continuity constraint. This means a call can be blocked even if there are free wavelengths (but not the same one) on all the links. Therefore, having full wavelength conversion is advantageous in that it decreases the blocking probability. However, implementing all-optical full wavelength conversion is quite difficult due to technological limitations. So, it is interesting to investigate whether we can do as well as full wavelength conversion in terms of blocking performance by using limited-range wavelength conversion, if not by using no wavelength conversion.

In limited-range wavelength conversion, an input wavelength can be converted to a limited range of outgoing wavelengths. Another form of limited wavelength conversion is to put a limit on the number of full wavelength converters provided to each node or to put a limit on the number of nodes that are provided full wavelength converters. In [7] Subramaniam et al. showed that the blocking performance remains almost the same if only a few nodes (as opposed to all) are equipped with full wavelength converters. They called this sparse wavelength conversion. Yates et al. [2] were the first to present results regarding limited-range wavelength conversion. They developed a simple analytical model for two-hop and multiple-hop paths and used 
simulations to analyze the performance of unidirectional ring and mesh-torus topologies. In [3] Sharma and Varvarigos presented an analytical model to compute blocking probabilities for mesh-torus networks with limited-range wavelength conversion and showed that limited wavelength conversion of degree 2 or 3 can give performance comparable to full wavelength conversion. But the analysis presented in [2], [3] was restricted to some specific network topologies.

In this paper, we develop an analytical model for computing blocking probabilities using limited-range wavelength conversion in all-optical networks. The model developed can be used for fixed routing and is applicable to any network topology. In this model we assume that a limited wavelength converter with conversion degree, $d$, can convert any incoming wavelength to $d$ adjacent wavelengths on either side, in addition to the input wavelength itself. Essentially, it means that any input wavelength can be switched to $(2 d+1)$ outgoing wavelengths. Using this model we quantify the benefit obtained using limitedrange wavelength conversion vis-a-vis no and full wavelength conversion. We are particularly interested to know whether we can achieve performance close to that of full wavelength conversion using limited-range wavelength conversion.

The rest of the paper is organized as follows. In section II, we present the analytical model for limited wavelength conversion. In section III we present the numerical results and in section IV we conclude.

\section{LIMITED-RANGE WAVELENGTH CONVERSION}

\section{A. Traffic Model}

There are many different models that have been used to describe the traffic demands for lightpaths between pairs of nodes and their use leads to substantially different performance optimization criteria for the network. Depending on the temporal nature of the demand for lightpaths, we can classify the lightpath requests into two categories: offline and online. In the offline case, we are given the entire set of lightpaths that are to be routed in advance. In the online case, the demands for lightpaths arise one at a time and each lightpath must be provided on demand without waiting for future lightpath demands to become known. Another important question is whether the network can block some lightpaths (the blocking model), or whether it must support all requested lightpaths (the nonblocking model) [11].

In our case, we consider the online blocking model. The lightpaths are set up and taken down on demand. These are analogous to setting up and taking down circuits in circuitswitched networks.

\section{B. Assumptions}

The following assumptions are used in our analytical model. 1. External calls arrive at each node according to an independent stationary Poisson process with rate $\lambda$.

2. Call holding time is exponentially distributed with unit mean.

3. Calls that cannot be routed in the network are blocked and lost.
4. The capacity of the links, denoted by $C$, is the same for all the links in the network. Each call requires a full wavelength on each link of its path.

5. Wavelengths are assigned uniformly randomly from the set of free wavelengths on the associated path.

6. Simplex connections are considered.

7. Existing lightpaths/calls cannot be reassigned different wavelengths to accommodate the new lightpath/call request.

\section{Analytical Model for Limited-Range Conversion}

We assume that for any given input wavelength, it is possible to translate it to a limited range of output wavelengths. More precisely, it is assumed that a wavelength can be converted to $d$ adjacent wavelengths on either side of the input wavelength, in addition to the input wavelength itself, where $d$ is the degree of conversion. Hence, any wavelength can be converted to $(2 d+1)$ wavelengths. For example, incoming wavelength $\lambda_{i}$ can be converted to any of the outgoing wavelengths $\lambda_{i-d}, \cdots, \lambda_{i}, \cdots, \lambda_{i+d}$. We also assume that the conversions are circularly symmetric.

Let $p_{m}\left(x_{1}, x_{2}, \cdots, x_{N}\right)$ denote the probability of having $m$ choices for the outgoing wavelengths on a $N$-hop path given that $x_{1}, \cdots, x_{N}$ wavelengths are free on links $1, \cdots, N$ respectively. If $\overline{\mathbf{x}}=\left(x_{1}, x_{2}, \cdots, x_{N}\right)$,

$$
p_{m}(\overline{\mathbf{x}})=\operatorname{Pr}\left[X_{R}=m \mid X_{1}=x_{1}, \cdots, X_{N}=x_{n}\right]
$$

where $X_{R}$ is a random variable denoting the number of choices for the outgoing wavelengths on route $R=\{1,2, \cdots, N\}$ and the random variables $X_{i}$ denote the number of free wavelengths on link $i$. Let us first consider the case of a two-hop route $R=$ $\{i, j\}$, for which

$$
p_{m}(x, y)=\operatorname{Pr}\left[X_{i, j}=m \mid X_{i}=x, X_{j}=y\right] .
$$

This is the probability of having $m$ possible outgoing wavelengths on a two link route given that $x$ and $y$ wavelengths are free on the first and the second link respectively. We can think of having a bipartite graph $(\mathbf{X}, \mathbf{Y})$, where the set of vertices $\mathbf{X}$ and $Y$ represent the set of wavelengths available on the first and second link respectively; hence the cardinality of both the sets is equal to the capacity of the link $C$, i.e., $|\mathbf{X}|=|\mathbf{Y}|=C$. Each vertex $u_{k} \in \mathbf{X}$ has an edge incident with the "facing" vertex $v_{k} \in \mathbf{Y}$ and $d$ adjacent vertices on either side of $v_{k}$ (see Fig. 1). Therefore, each vertex of $\mathrm{X}$ will have a degree of $(2 d+1)$. The $(2 d+1)$ vertices in set $\mathbf{Y}$ which are adjacent to a vertex in set $\mathbf{X}$ are called its neighbours. For example, the neighbours of vertex $2 \in \mathbf{X}$ are vertices 1,2 and $3 \in \mathbf{Y}$ as shown in the Fig. 1 . The edges denote the possible conversion from one wavelength to another wavelength. For the last (resp. first) vertex, we will have edges to the immediately higher (resp. lower) $d$ vertices and the first (resp. last) $d$ vertices from the top (resp. bottom), i.e., the wavelength conversion is assumed to be circularly symmetric. This is merely for the sake of analytical convenience, as it distributes the load uniformly among all the wavelengths on a link. Let $\mathbf{X}_{i} \subseteq \mathbf{X}$ and $\mathbf{Y}_{j} \subseteq \mathbf{Y}$ denote those vertices corresponding to which we have free wavelengths on link $i$ and link $j$ respectively. The cardinality of sets $\mathbf{X}_{i}$ and $\mathbf{Y}_{j}$ are $x$ 


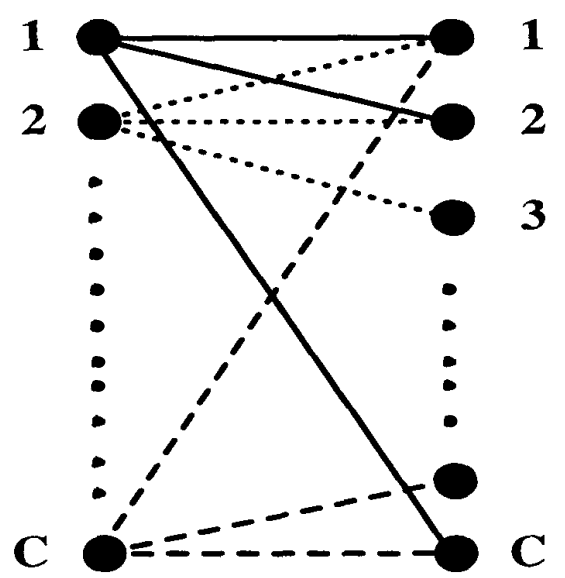

Fig. 1. Possible wavelength conversions at each node for $d=1$. The conversions are circularly symmetric.

and $y$, which is the number of free wavelengths on link $i$ and $j$ respectively.

Let $\Gamma\left(\mathbf{X}_{i}\right)$ denote the neighbours of the vertices in set $\mathbf{X}_{i}$. Then we are interested in finding the probability of having $m$ such neighbours of vertices in $\mathbf{X}_{i}$ which are incident with the vertices in $\mathbf{Y}_{j}$. Then

$$
\begin{aligned}
p_{m}(x, y)= & \operatorname{Pr}\left[\left|\Gamma\left(\mathbf{X}_{i}\right) \cap \mathbf{Y}_{j}\right|=m\right] \\
= & \sum_{l:\left|\Gamma\left(\mathbf{X}_{i}\right)\right|=l,\left|\mathbf{X}_{i}\right|=x} \frac{\left(\begin{array}{c}
\left|\Gamma\left(\mathbf{X}_{i}\right)\right| \\
m
\end{array}\right)\left(\begin{array}{c}
C-\left|\Gamma\left(\mathbf{X}_{i}\right)\right| \\
y-m
\end{array}\right)}{\left(\begin{array}{l}
C \\
y
\end{array}\right)} \\
& \times \operatorname{Pr}\left(\left|\Gamma\left(\mathbf{X}_{i}\right)\right|=l|| \mathbf{X}_{i} \mid=x\right) \\
= & \sum_{l=\min [C, x+2 d]}^{\min [C,(2 d+1) x]} \frac{\left(\begin{array}{c}
l \\
m
\end{array}\right)\left(\begin{array}{c}
C-l \\
y-m
\end{array}\right)}{\left(\begin{array}{l}
C \\
y
\end{array}\right)} \\
& \times \operatorname{Pr}\left(\left|\Gamma\left(\mathbf{X}_{i}\right)\right|=l|| \mathbf{X}_{i} \mid=x\right)
\end{aligned}
$$

The last equality in (3) has the summation running from $\min [C,(x+2 d)]$ to $\min [C,(2 d+1) x]$. This is because the minimum cardinality (neighbours) of $\Gamma\left(\mathbf{X}_{i}\right)$ will be $(x+2 d)$ or $C$, depending on whichever is smaller, and the maximum cardinality (neighbours) can be either $C$ or $(2 d+1) x$, depending on whichever is smaller, as the number of neighbours can't be more than $C$ (the capacity of the link).

When there is only one wavelength (vertex) free belonging to the set $\mathbf{X}_{i}$, i.e., $\left|\mathbf{X}_{i}\right|=1$, then the number of neighbours of that vertex will be exactly $(2 d+1)$ and in this case the lower and upper limits of summation coincide. In the case when one has exactly $x$ wavelengths (vertices) free in the set $\mathbf{X}_{i}$, i.e., $\left|\mathbf{X}_{i}\right|=x$, and if all the $x$ wavelengths are adjacent then we have only $\min [(x+2 d), C]$ distinct neighbours. This one extreme case constitutes the lower limit. Had all these $x$ free wavelengths (vertices) been sufficiently apart from one another so that each contributes $(2 d+1)$ distinct neighbours then we will have $(2 d+1) x$ distinct neighbours provided $(2 d+1) x$ is less than $C$; otherwise this number will be $C$. This other extreme case constitutes the upper limit of the summation. For all other cases the number of neighbours lies between these two limits.

Now, for the case when $m=0$, we have

$$
\begin{aligned}
p_{0}(x, y)= & \sum_{l=\min [C, x+2 d]}^{\min [C,(2 d+1) x]} \frac{\left(\begin{array}{c}
C-l \\
y
\end{array}\right)}{\left(\begin{array}{l}
C \\
y
\end{array}\right)} \times \\
& \operatorname{Pr}\left(\left|\Gamma\left(\mathbf{X}_{i}\right)\right|=l|| \mathbf{X}_{i} \mid=x\right) .
\end{aligned}
$$

Note that in general $p_{m}(x, y) \neq p_{m}(y, x)$ but interestingly, it can be shown that $p_{0}(x, y)=p_{0}(y, x)^{\prime}$. When $d=0$, our model reduces to Birman's model [4] for no wavelength conversion. In this case, $p_{m}(x, y)=p_{m}(y, x)$, by symmetry. We can rearrange the links such that the links of the path have free wavelengths in increasing order and then we can use (3) to compute $p_{m}(x, y)$.

The probability term, $\operatorname{Pr}($.$) in (3) and (4) is given by$

$$
\begin{aligned}
\operatorname{Pr}\left[\left|\Gamma\left(\mathbf{X}_{i}\right)\right|\right. & \left.=l|| \mathbf{X}_{i} \mid=x\right] \\
= & \operatorname{Pr}\left[\left|\Gamma\left(\mathbf{X}_{i}\right)\right| \leq l|| \mathbf{X}_{i} \mid=x\right] \\
& \quad-\operatorname{Pr}\left[\left|\Gamma\left(\mathbf{X}_{i}\right)\right| \leq l-1|| \mathbf{X}_{i} \mid=x\right] .
\end{aligned}
$$

First note that $\operatorname{Pr}\left[\left|\Gamma\left(\mathbf{X}_{i}\right)\right| \leq l|| \mathbf{X}_{i} \mid=x\right]=1$ if $l=C$ or $l=(2 d+1) x$ since the number of neighbours cannot exceed either number. Also $\operatorname{Pr}\left[\left|\Gamma\left(\mathbf{X}_{i}\right)\right| \leq l|| \mathbf{X}_{i} \mid=x\right]=0$ if $l<$ $x+2 d \leq C$ since there are at least $x+2 d$ neighbours, in this case. Hence, for the remainder of the discussion, we assume $x+2 d \leq l<\min [C,(2 d+1) x]$.

Consider the probability that $\left|\Gamma\left(\mathbf{X}_{i}\right)\right| \leq l$, given that the vertices $\Gamma\left(\mathbf{X}_{i}\right)$ lie in some contiguous range of $l$ vertices (and $\left|\mathbf{X}_{i}\right|=x$ ). In this range some of the vertices may not be neighbours of $\mathbf{X}_{i}$. Therefore, the total number of neighbours is at most $l$. Clearly there are cases when we have no more than $l$ neighbours but they do not lie in some contiguous range of $l$ vertices. This yields the inequality

$$
\begin{aligned}
& \operatorname{Pr}\left[\left|\Gamma\left(\mathbf{X}_{i}\right)\right| \leq l|| \mathbf{X}_{i} \mid=x\right] \geq \\
& \operatorname{Pr}\left[\left|\Gamma\left(\mathbf{X}_{i}\right)\right| \leq l, l \text { contiguous }|| \mathbf{X}_{i} \mid=x\right] .
\end{aligned}
$$

We have,

$$
\operatorname{Pr}\left[\left|\Gamma\left(\mathbf{X}_{i}\right)\right| \leq l, l \text { contiguous }\right]=\operatorname{Pr}\left[\cup_{k=1}^{C} \Gamma\left(\mathbf{X}_{i}\right) \subseteq l_{k}\right]
$$

where each $l_{k}$ is a set of contiguous vertices of size $l$ and $k$ varies from 1 to $C$ as we can position $l$ contiguous vertices in $C$ ways because the conversion is assumed to be circularly symmetric.

\footnotetext{
${ }^{1} p_{0}(x, y)$ is the probability that $\Gamma\left(X_{i}\right) \cap Y_{j}=\phi$. We will prove that whenever $\Gamma\left(X_{i}\right) \cap Y_{j}=\phi, \Gamma\left(Y_{j}\right) \cap X_{i}=\phi$. We prove this by contradiction. Suppose $\Gamma\left(Y_{j}\right) \cap X_{i} \neq \phi$. Then there exists a vertex $u_{i} \in X_{i}$ s.t. $u_{i} \in \Gamma\left(Y_{j}\right) \cap X_{i}$. Since $u_{i} \in \Gamma\left(Y_{j}\right)$, there exists a vertex $v_{j} \in Y_{j}$ s.t. $\Gamma\left(v_{j}\right)=u_{i}$. Therefore, $\Gamma\left(u_{i}\right)=v_{j}$ which contradicts $\Gamma\left(X_{i}\right) \cap Y_{j}=\phi$.
} 


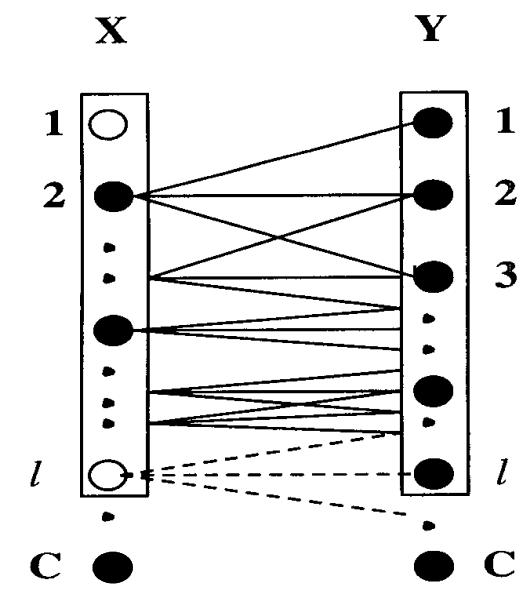

Fig. 2. This figure is for limited conversion with degree $d=1$. If all the neighbours must lie within a contiguous range of $l$ vertices, we cannot choose the first and last $d$ vertices from the set $\mathbf{X}$. Here we cannot choose the first and the $l$ th vertices shown by the hollow circles. For example if we choose the $l$ th vertex its neighbours falls outside the range $l$ as shown in the figure above.

Let us denote the event $\Gamma\left(\mathbf{X}_{i}\right) \subseteq l_{k}$ by $Z_{k}$ (given that $\left|\mathbf{X}_{i}\right|=$ $x$ ). Then the R. H. S. of (6) is given by

$$
\begin{aligned}
\operatorname{Pr}\left[\cup_{k=1}^{C} \Gamma\left(\mathbf{X}_{i}\right) \subseteq l_{k}\right] & =\operatorname{Pr}\left[Z_{1} \text { or } Z_{2} \text { or } \cdots \text { or } Z_{C}\right] \\
& \leq\left(\begin{array}{l}
C \\
1
\end{array}\right) \operatorname{Pr}\left[Z_{k}\right]
\end{aligned}
$$

because we can choose $l$ contiguous vertices in $\left(\begin{array}{l}C \\ 1\end{array}\right)$ ways. Now consider one such contiguous set of $l$ vertices in the set $\mathbf{Y}_{j}$, say set $\mathbf{L}_{j}$. As per our assumption the $x$ vertices in set $\mathbf{X}$ are contained in the "facing" vertices of the vertices in set $\mathbf{L}_{j}$. We denote this set of "facing" vertices as set $\mathbf{X}_{l}$ which has cardinality $l$. We want to choose $x$ vertices in $\mathbf{X}_{l}$ such that their neighbours are in set $\mathbf{L}_{j}$. If any vertex is in the first $d$ vertices or the last $d$ vertices of the set $\mathbf{X}_{l}$, some neighbours must fall outside the range of set $\mathbf{L}_{j}$ as shown in Fig. 2. Therefore $x$ vertices can be chosen only from $(l-2 d)$ vertices of set $\mathbf{X}_{l}$. The total number of ways in which set $\mathbf{X}_{i}$, such that $\left|\mathbf{X}_{i}\right|=x$, can be formed is $\left(\begin{array}{l}C \\ x\end{array}\right)$. Therefore, the probability that $\Gamma\left(\mathbf{X}_{i}\right) \mid \subseteq l_{k}$, when $l_{k}$ is a set of contiguous vertices of size $l$, is given by

$$
\operatorname{Pr}\left[Z_{k}\right]=\frac{\left(\begin{array}{c}
l-2 d \\
x
\end{array}\right)}{\left(\begin{array}{l}
C \\
x
\end{array}\right)}
$$

Therefore, from (6), (7) and (8), we get

$$
\operatorname{Pr}\left[\left|\Gamma\left(\mathbf{X}_{i}\right)\right| \leq l, l \text { contiguous }\right] \leq \frac{\left(\begin{array}{l}
C \\
1
\end{array}\right)\left(\begin{array}{c}
l-2 d \\
x
\end{array}\right)}{\left(\begin{array}{l}
C \\
x
\end{array}\right)}
$$

We have already mentioned that $\operatorname{Pr}\left[\left|\Gamma\left(\mathbf{X}_{i}\right)\right| \leq l\right.$ is lower bounded by the L. H. S. of (9) given that $\left|\mathbf{X}_{i}\right|=x$. Thus this does not yield a bound on $\operatorname{Pr}\left[\left|\Gamma\left(\mathbf{X}_{i}\right)\right| \leq l\right.$. We assume the following approximation holds for $x+2 d \leq l<$ $\min [C,(2 d+1) x]:$

$$
\operatorname{Pr}\left[\left|\Gamma\left(\mathbf{X}_{i}\right)\right| \leq l|| \mathbf{X}_{i} \mid=x\right] \approx \frac{\left(\begin{array}{c}
C \\
1
\end{array}\right)\left(\begin{array}{c}
l-2 d \\
x
\end{array}\right)}{\left(\begin{array}{l}
C \\
x
\end{array}\right)} .
$$

We will see from numerical results later that this yields a good approximation to the blocking probabilities, at least for small values of the conversion degree $d$.

For the general case of an $N$-hop route, $N \geq 3$, let $x_{j}$ be the number of the idle wavelengths on the $j$ th hop. We condition on the set of disjoint events $\left\{X_{\bar{R}}=k \mid k=m, x_{1}, x_{2}, \cdots x_{N-1}\right\}$, where $\bar{R}=\{1, \cdots, N-1\}$. Recall that $X_{R}$ is a random variable denoting the number of possible outgoing wavelengths on route $R$. We thus obtain the recursive relation (assume the first $(N-1)$ links to be the first link and the last link to be the second link):

$$
p_{m}\left(x_{1}, \cdots, x_{N}\right)=\sum_{k=m}^{x_{N-1}} p_{k}\left(x_{1}, \cdots, x_{N-1}\right) p_{m}\left(k, x_{N}\right)
$$

where $p_{m}\left(k, x_{N}\right)$ is given by (3).

\section{Fixed Wavelength Routing}

We consider a network with an arbitrary topology with $J$ links and $C$ wavelengths on each link. A route $R$ is a subset of links $\{1, \cdots, J\}$. Calls arrive for route $R$ as a Poisson stream with rate $a_{R}$. An incoming call on route $R$ is set up if it finds a free wavelength on all the links from the possible choices of outgoing wavelengths with the given degree of limited wavelength conversion. If such a combination of wavelengths is not possible on the links constituting the path, then the call is blocked and lost. If the call is accepted it simultaneously holds the wavelength/wavelengths on all the links on route $R$ for the duration of the call. The holding times of all the calls are assumed to be exponentially distributed with unit mean.

Let $X_{j}$ be the random variable denoting the number of idle wavelengths on link $j$ in equilibrium. Let $\mathbf{X}=\left(X_{1}, \cdots, X_{J}\right)$ and let

$$
q_{j}(w)=\operatorname{Pr}\left[X_{j}=w\right] ; \quad w=0, \cdots, C
$$

be the idle capacity distribution. Throughout the following approximations are made:

1. The random variables $X_{1}, X_{2}, \cdots, X_{J}$ are mutually independent. Then

$$
q(\mathbf{w})=\prod_{j=1}^{J} q_{j}\left(w_{j}\right)
$$

where $\mathbf{w}=\left(w_{1}, w_{2}, \cdots, w_{J}\right)$

2. When there are $w$ idle wavelengths on link $j$, the time until the next call is set up on link $j$ is exponentially distributed with parameter $\alpha_{j}(w)$. This parameter is the call set up rate on link $j$ when $w$ wavelengths are free on link $j$. 
From the approximation (2), it follows that the number of idle wavelengths on link $j$ can be viewed as a birth-and-death process and therefore we have

$$
\begin{array}{r}
q_{j}(w)=\frac{C(C-1) \cdots(C-w+1)}{\alpha_{j}(1) \alpha_{j}(2) \cdots \alpha_{j}(w)} q_{j}(0), \\
w=1, \cdots, C,
\end{array}
$$

where

$$
q_{j}(0)=\left[1+\sum_{w=1}^{C} \frac{C(C-1) \cdots(C-w+1)}{\alpha_{j}(1) \alpha_{j}(2) \cdots \alpha_{j}(w)}\right]^{-1} .
$$

The call set up rate on link $j$ when there are $w$ idle wavelengths on link $j, \alpha_{j}(w)$, is obtained by combining the contributions from the request streams to routes of which link $j$ is a member.

$$
\begin{gathered}
\alpha_{j}(w)=0, \quad \text { if } \quad w=0, \\
=\sum_{R: j \in R} a_{R} \operatorname{Pr}\left[X_{R}>0 \mid X_{j}=w\right], \\
w=1, \cdots, C .
\end{gathered}
$$

If the route consists of a single link then the probability term $\operatorname{Pr}($.$) under the summation sign in (13) will be equal to 1$. If the route consists of two links, let $R=\{i, j\}$. The term $\operatorname{Pr}($. can be further simplified by conditioning it on the set of disjoint events $\left\{X_{i}=l \mid l=0, \cdots, C\right\}$.

$$
\begin{aligned}
& \operatorname{Pr}\left[X_{\{i, j\}}>0 \mid X_{j}=w\right] \\
= & \sum_{l=1}^{C} \operatorname{Pr}\left[X_{i}=l \mid X_{j}=w\right] \operatorname{Pr}\left[X_{R}>0 \mid X_{j}=w, X_{i}=l\right] \\
= & \sum_{l=1}^{C} \operatorname{Pr}\left[X_{i}=l\right]\left(1-\operatorname{Pr}\left[X_{R}=0 \mid X_{j}=w, X_{i}=l\right]\right. \\
= & \sum_{l=1}^{C} q_{i}(l)\left(1-p_{0}(w, l)\right),
\end{aligned}
$$

where $p_{0}(w, l)$ is given by (4). The second equality above is obtained by using the assumption that the random variables $X_{i}$ are independent. Similarly, for a three hop route $R=\{i, j, k\}$ we obtain:

$\operatorname{Pr}\left[X_{\{i, j, k\}}>0 \mid X_{j}=w\right]=\sum_{l=1}^{C} \sum_{n=1}^{C} q_{i}(l) q_{k}(n)\left[1-p_{0}(l, w, n)\right]$

where $p_{0}(l, w, n)$ is obtained from (10) and (3).

\section{E. Computation of Blocking Probability}

The blocking probability for calls to route $R$ is

$$
\begin{aligned}
L_{R} & =\operatorname{Pr}\left[X_{R}=0\right] \\
& =q_{i}(0), \quad \text { if } \quad R=\{i\} \\
& =\sum_{l=0}^{C} \sum_{w=0}^{C} q_{i}(l) q_{j}(w) p_{0}(l, w), \quad \text { if } \quad R=\{i, j\} \\
& =\sum_{l=0}^{C} \sum_{w=0}^{C} \sum_{n=0}^{C} q_{i}(l) q_{j}(w) q_{k}(n) p_{0}(l, w, n) \\
& \quad \text { if } \quad R=\{i, j, k\}
\end{aligned}
$$

\section{F. Algorithm for Computation of Blocking Probability}

The algorithm below uses a fixed-point method to compute the approximate blocking probabilities for the traffic on all the routes and the (average) blocking probability of the network.

1. Initialization. For all the routes $R$ let $\hat{L}_{R}=0$. For $j=$ $1, \cdots, J$, let $\alpha_{j}(0)=0$, and let $\alpha_{j}(m)$ be chosen arbitrarily, $m=1, \cdots, C$.

2. Determine $q_{j}($.$) from (11) and (12).$

3. Obtain new values of $\alpha_{j}(),. j=1, \cdots, J$, using (13). (Note that (14) must be used in (13) for 2-hop paths, (15) in (13) for 3-hop paths, and suitable generalizations for paths with more hops.)

4. Calculate $L_{R}$, for all routes $R$, using (16). If $\max _{R} \mid L_{R}-$ $\hat{L}_{R} \mid<\epsilon$ (where $\epsilon$ is suitably small positive quantity), then terminate. Otherwise let $\hat{L}_{R}=L_{R}$, and go to step 2 .

5 . The (average) blocking probability of the network is then given by

$$
P b_{\text {network }}=\frac{\sum_{r=1}^{R} a_{r} L_{r}}{\sum_{r=1}^{R} a_{r}} .
$$

\section{NUMERICAL RESULTS}

We present simulation and analytical results for an example network with 6 nodes and 7 links (Fig. 3), a 6 node ring network (Fig. 4) and the NSFNET backbone (Fig. 5) for three different cases: no wavelength conversion, limited wavelength conversion with degree $d=1,2$ (and 3 for the NSFNET), and full wavelength conversion. For first two networks we consider connections between all possible node pairs, so that the number of possible routes is 15 . For the NSFNET, we have taken 71 routes. (These are the routes for which the shortest hop path is unique.) The offered traffic on each route is assumed to be equal (uniform traffic) and we plot the (average) blocking probability (over all routes) versus the total offered load to the network. We observe that in all the networks limited conversion provides a considerable fraction of the improvement that full wavelength conversion provides over no wavelength conversion.

In simulations, for the no wavelength conversion case, we first find the wavelengths which are free on all the links of the path. We then choose a wavelength out of these free wavelengths uniformly randomly and assign it to the new call. If no such wavelength is available then the new call is blocked.

For full wavelength conversion, we choose a wavelength out of the free wavelengths on the first hop uniformly randomly and do the same on the subsequent hops of the path. If there is no wavelength free on some link of the path then the new call is blocked.

For limited wavelength conversion, we choose a wavelength out of the free wavelengths on the first hop uniformly randomly and at each subsequent hop look for the possible outgoing wavelengths with the given degree of wavelength conversion. If more than one such wavelength is available then once again we choose a wavelength uniformly randomly on this hop. This is repeated on subsequent hops. If at some hop (other than the 
first), there is no wavelength free which is in the possible subset of outgoing wavelengths then we fall back to the previous hop and choose a wavelength out of the free wavelengths minus the earlier chosen free wavelength/wavelengths uniformly randomly. If we exhaust all the free wavelengths on the first hop and still cannot find any possible outgoing wavelength on some hop then we block the call.

For full wavelength conversion and no wavelength conversion, we have used the reduced load approximation given in [9] and Birman's method [4] respectively to calculate the blocking probability analytically.

For the 6 node example network (Fig. 3), we plot the graphs for 16 wavelengths showing the performance of full, no and limited wavelength conversion. From Fig. 6, we see that the performance obtained by limited wavelength conversion with degree $d=1$ is close to the performance of full conversion and with degree $d=2$ it almost matches the full wavelength conversion performance.

In Fig. 7, we show that our analysis results for limited wavelength conversion with degree $d=1$ are in good agreement with the results of simulations. For blocking probabilities of the order of $10^{-3}$ and above, the percentage difference is well within $5 \%$.

In Fig. 8, we plot the curves for 16 wavelengths for a ring network. Again the limited wavelength conversion performance is very close to that of full wavelength conversion. In fact, for the 6 node ring network, with conversion degree $d=2$, we can virtually achieve the same performance as that of full conversion as shown in Fig. 8. In Fig. 9 we compare the results of our analysis and simulations for $d=2$ and see that they match quite well.

In Fig. 10, we plot the curves for 12 wavelengths for the NEFNET backbone network of Fig. 5. Again the limited wavelength conversion performance is very close to that of full wavelength conversion. We also note from Fig. 11 that our analysis is quite accurate for $d=3$.

We have presented three examples to show the benefits of limited-range wavelength conversion, and in all these examples, limited conversion provides a marked improvement in the blocking performance of the network as compared to no wavelength conversion. Furthermore the performance obtained by limited conversion with small values of the conversion degree, such as $d=1$ or $d=2$, is very close to the blocking performance of the network with full wavelength conversion.

The computational requirements of the wavelength routing model with limited wavelength conversion presented in previous section are significant: exponential in terms of the number of hops. The complexity of calculating the blocking probability $L_{R}$ is of the order of $O\left(C^{H}\right)$, where $H$ denotes the number of hops of route $R$. The technique of truncated distribution [8] could be applied here as well, and will alleviate the problem somewhat for moderate and heavy traffic. It may also be possible to develop a parallel implementation of the fixed point equations proposed in this paper for limited wavelength conversion, along the lines of the approach presented in [12] for circuit-switched (i.e., full wavelength conversion) networks.

\section{CONCLUSIONS}

In this paper, we have proposed a method to calculate the average blocking probability in optical networks using limitedrange wavelength conversion. The proposed analytical model in this paper is applicable to any topology. Using this model we have demonstrated that the performance improvement obtained by full wavelength conversion over no wavelength conversion can be achieved by using limited wavelength conversion with the degree of conversion, $d$, being only 1 or 2 .

We have also seen that our analytical model for limited wavelength conversion is quite accurate for small but non-zero values of the conversion degree $d$. For $d=0$ our model reduces to that of Birman [4] for the case of no wavelength conversion and the analytical values are not as accurate in this case. We conjecture that this is because correlations between the use of wavelengths on the links are quite significant in the case when $d=0$ while our model assumes link independence. For higher values of $d$, the correlations probably decrease. We are exploring how correlations in wavelength usage on different links can be taken into account to improve the accuracy of our analytical method.

Finally, we have only considered routes with up to 3 -hops and confined ourselves to fixed routing. We need to take longer routes than this to further explore the benefits of limited-range wavelength conversion. The model presented in this paper, especially that used for computing the $p_{m}($.$) , can also be used for$ alternate routing with limited-range wavelength conversion by extending the method presented in [5]. We are exploring this further.

\section{REFERENCES}

[1] R. Ramaswami and K. N. Sivarajan. Routing and Wavelength Assignment in All-Optical Networks. IEEE/ACM Transactions on Networking. 3(5):489-500, October 1995.

[2] J. Yates, J. Lacey, D. Everitt, and M. Summerfield. Limited-range wavelength translation in all-optical networks. IEEE INFOCOM, 954-961, 1996.

[3] V. Sharma and E. A. Varvarigos. Limited wavelength translation in alloptical WDM mesh networks IEEE INFOCOM, 893-901, 1998.

[4] A. Birman. Computing approximate blocking probabilities for a class of all-optical networks. IEEE JSAC/JLT Special Issue on Optical Networks. 14(5): $852-857$, June 1996

[5] H. Harai, M. Masayuki, and H. Miyahara. Performance of alternate routing methods in all-optical switching networks IEEE INFOCOM, 1997.

[6] R. A. Barry and P. A. Humblet. Model of blocking probability in all optical networks with and without wavelength changers. IEEE INFOCOM. 402-412, April 1995.

[7] S. Subramaniam, M. Azizoglu and A. K. Somani. All-optical networks with sparse wavelength conversion. IEEE/ACM Transactions on Net working., 4(4):544-557, Aug. 1996.

[8] S. P. Chung, A. Kashper and K. W. Ross. Computing approximate blocking probabilities for large loss network with state-dependent routing. IEEE/ACM Transactions on Networking., 1(1):105-115, Feb. 1993

[9] S. P. Chung and K. W. Ross. Reduced load approximations for multirate loss networks. IEEE Transactions on Communications., 41(8): 1222 1231, Aug. 1993.

[10] M. Kovacevic and A. Acampora. Benefits of wavelength translation in all-optical clear-channel networks. IEEE Journal of Selected Areas in Communications., 14(5): 868-880, June 1996.

[11] R. Ramaswami and K. N. Sivarajan. Optical Networks: A Practical Perspective, Morgan Kaufmann Publishers, San Francisco, 1998.

[12] A. G. Greenberg and R. Srikant. Computational techniques for accurate performance evaluation of multirate, multihop communication networks. IEEE/ACM Transaction on Networking, 5(2): 266-277, April 1997. 


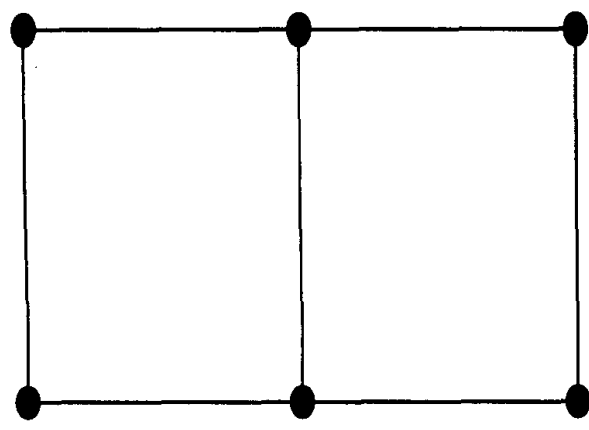

Fig. 3. An example network with 6 nodes and 7 links.

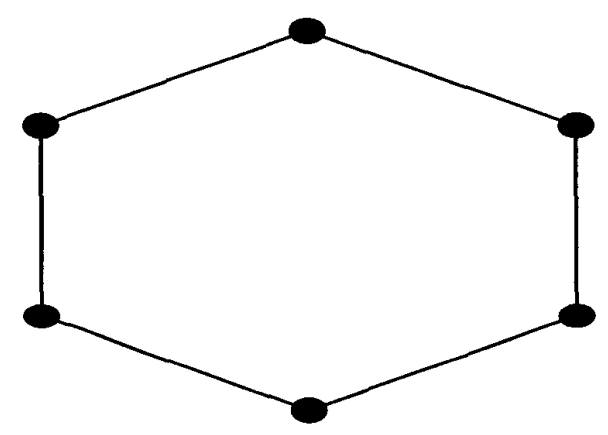

Fig. 4. Six node ring network.

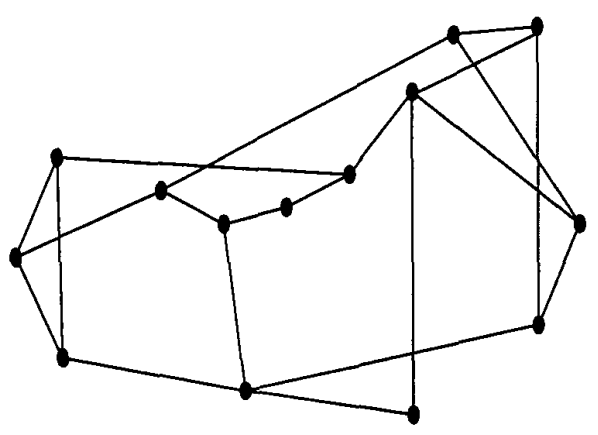

Fig. 5. NSFNET backbone consisting of 14 nodes and 21 links.

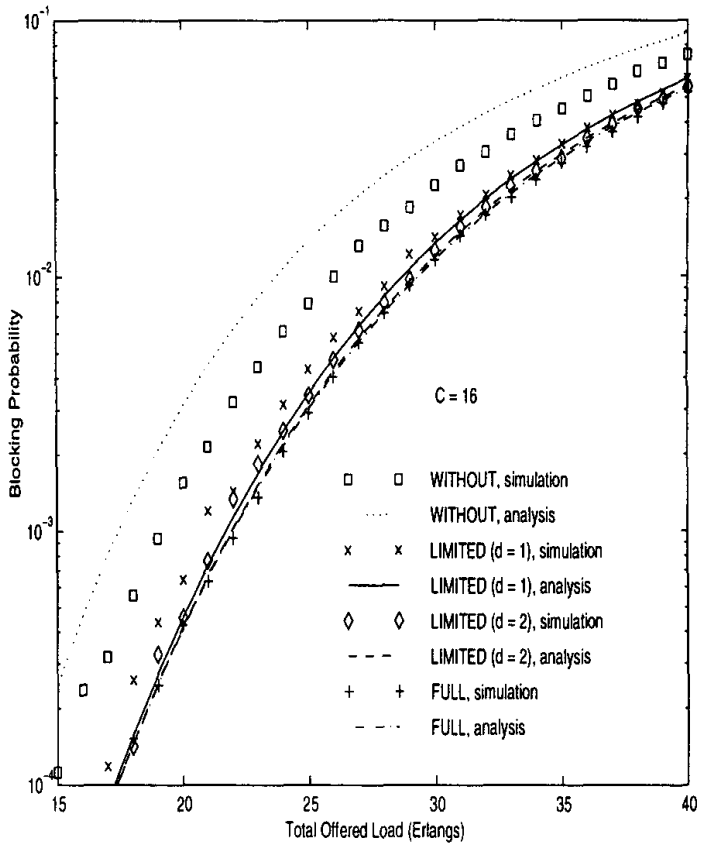

Fig. 6. The average blocking probability in the example network with 6 nodes and 7 links versus the total offered load, for $C=16$ wavelengths per link. The plot shows the analytically calculated values and simulation values for no, full and limited wavelength conversion with conversion degree $d=1$ and $d=2$.

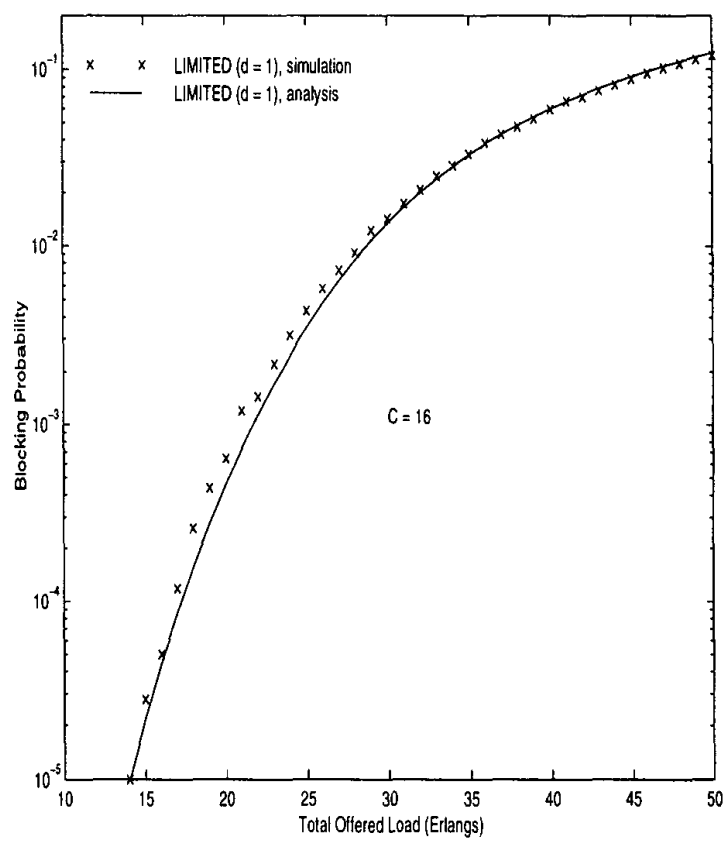

Fig. 7. Comparison of the results obtained through analysis and simulation for limited wavelength conversion with degree $d=1$ for the example network with 6 node and 7 links. 


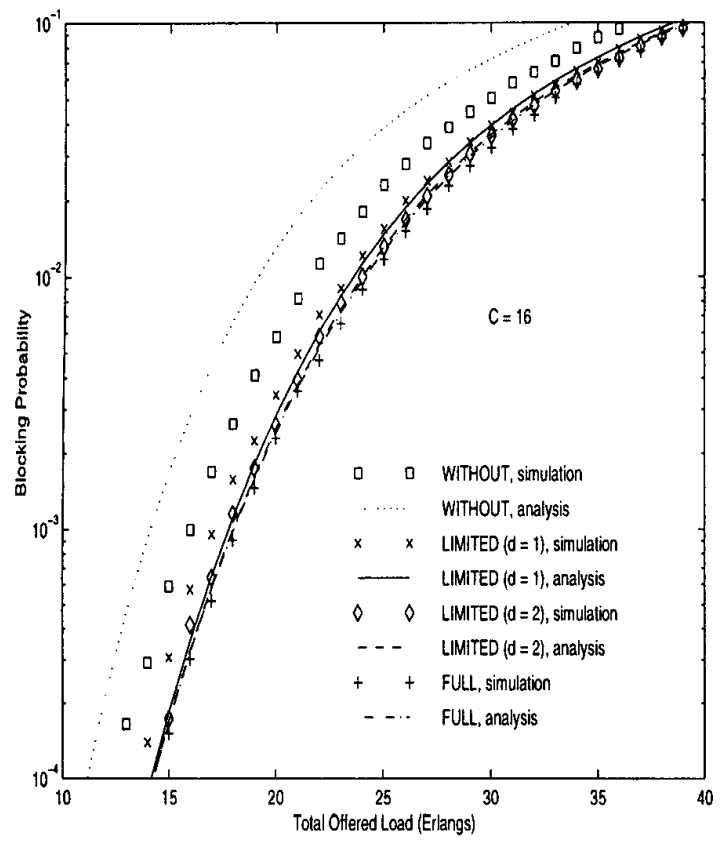

Fig. 8. The average blocking probability in the 6 node ring network versus the total offered load, for $C=16$ wavelengths per link. The plot shows the analytically calculated values and simulation values for no, full and limited wavelength conversion with conversion degree $d=1$ and $d=2$.

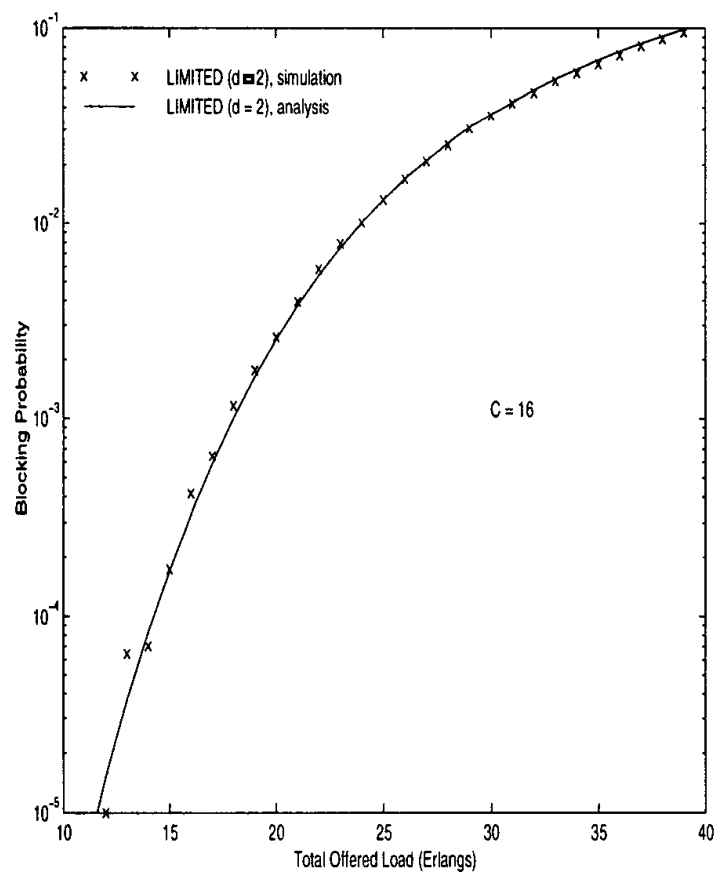

Fig. 9. Comparison of the results obtained through analysis and simulation for limited wavelength conversion with degree $d=2$ for the 6 node ring network.

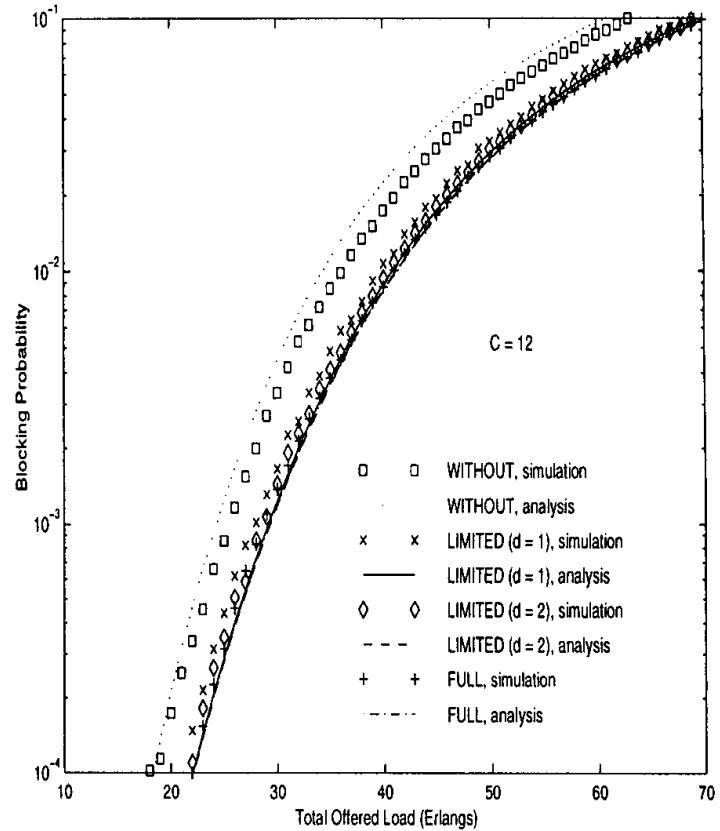

Fig. 10. The average blocking probability in the NSFNET network versus the total offered load, for $C=16$ wavelengths per link. The plot shows the analytically calculated values and simulation values for no, full and limited wavelength conversion with conversion degree $d=1$ and $d=2$.

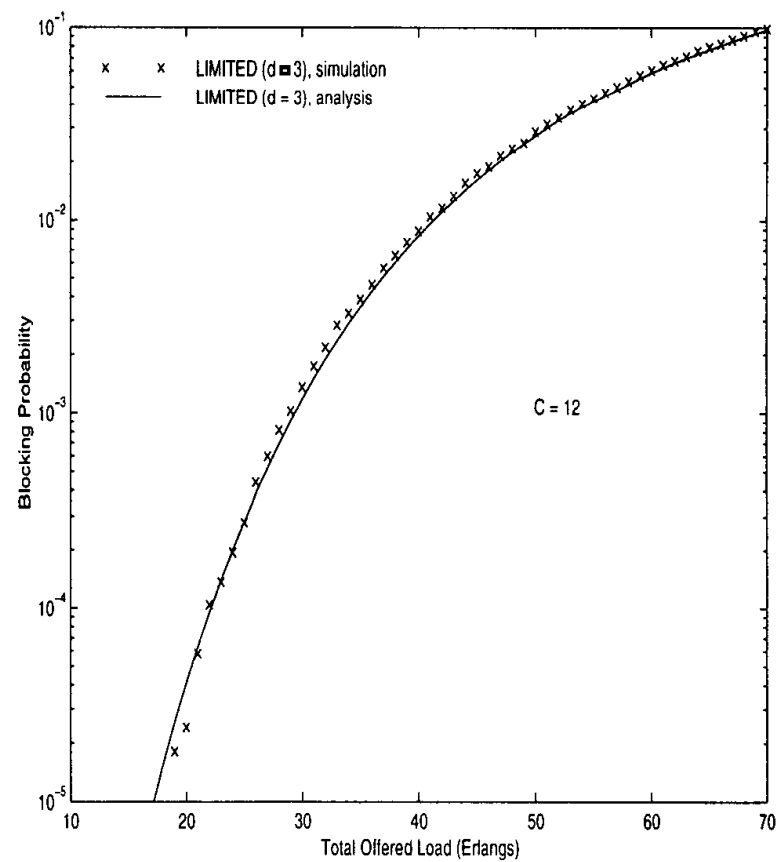

Fig. 11. Comparison of the results obtained through analysis and simulation for limited wavelength conversion with degree $d=3$ for the NSFNET. 